\title{
Hepatitis C Direct-Acting Antiviral Treatment Selection, Treatment Failure, and Use of Drug-Drug Interactions in a State Medicaid Program
}

\author{
Shellie L. Keast, PharmD, PhD; Bethany Holderread, PharmD; Terry Cothran, DPh; \\ and Grant H. Skrepnek, PhD
}

\begin{abstract}
BACKGROUND: Newer hepatitis C virus (HCV) treatments often provide high success rates with fewer adverse events, although the extent of all potential drug interactions is not fully known.

OBJECTIVE: To assess outcomes of receiving HCV treatment and subsequent sustained virologic response (SVR) based on patient and clinical characteristics, including direct-acting antiviral (DAA) drug-drug interactions (DDIs), in Medicaid members with chronic HCV.

METHODS: Comprehensive medical and pharmacy claims and prior authorization data were collected for HCV patients requesting treatment between January 2014 and June 2015. Outcomes of receiving treatment with DAAs and treatment failure based on SVR were analyzed according to demographics, prior/current HCV treatment, severity of DDls, advancing liver disease, and comorbidities. Multivariable generalized linear models were employed, including a Bayesian sensitivity analysis.
\end{abstract}

RESULTS: Among 3,412 Medicaid members with HCV, 13.6\% received DAAs $(n=464)$, averaging $53.6 \pm 10.0$ years, with $52.8 \%$ female. Multivariable analyses indicated that higher odds of DAA treatment initiation were associated with older age, prior HCV treatment, and advancing liver disease. Some $4.8 \%$ of treatment failures occurred among 168 patients with reported SVRs, wherein a 3.218 times higher adjusted odds of treatment failure was associated with concomitant use of medications with DDIs classified as significant or potentially clinically significant by the University of Liverpool HEP Drug Interactions resource $(P=0.001)$.

CONCLUSIONS: In a cohort of state Medicaid members with chronic HCV, a markedly higher adjusted odds of treatment failure was independently associated with DDIs classified as significant or potentially clinically significant, warranting continued inquiry and potential alternate treatments concerning conditions that require their use.

J Manag Care Spec Pharm. 2019;25(11):1261-67

Copyright @ 2019, Academy of Managed Care Pharmacy. All rights reserved.

\section{What is already known about this subject}

Chronic hepatitis $\mathrm{C}$ is a bloodborne viral infection whose sequelae involves chronic liver disease, cirrhosis, and liver cancer and affects an estimated 2.7 million persons in the United States. Oral direct-acting antivirals (DAAs) indicated for the treatment of HCV achieve high cure rates across multiple HCV genotypes. Concomitant medications classified to interact with DAAs by the University of Liverpool HEP Drug Interactions resource may help establish the interactions that potentially affect cure rates.

\section{What this study adds}

This study assessed the outcomes of receiving HCV treatment and subsequent sustained virologic response based on patient and clinical characteristics, including DAA drug-drug interactions (DDIs), in Medicaid members with chronic HCV.

Potential predictors of HCV treatment failure with DAAs warrant further evaluation, particularly in specific populations and among those receiving multiple medications with the potential to interact negatively with DAA therapies.

Patients with chronic HCV had markedly higher odds of treatment failure if using concomitant medications with DDIs classified as significant or potentially clinically significant, warranting continued inquiry and potential alternate treatments concerning conditions that require their use.

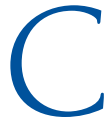
hronic hepatitis $\mathrm{C}(\mathrm{CHC})$ is a bloodborne viral infection that affects an estimated 2.7 million persons in the United States. If If untreated, individuals infected with hepatitis $\mathrm{C}$ virus (HCV) can develop chronic liver disease, cirrhosis, liver cancer, and death. ${ }^{2} \mathrm{HCV}$ infection is most prevalent among persons born between 1945 and 1965, and a national health survey found infected persons were more likely to have less education and lower family income., ${ }^{1,3}$

HCV treatment has evolved quickly in recent years. Before 2011, HCV was treated with pegylated interferon and ribavirin combination therapies, achieved sustained virologic response (SVR) rates of approximately 50\%, and had high occurrences of side effects. ${ }^{2,4}$ Beginning in 2011, improved cure rates followed the first generation of oral direct-acting antivirals (DAAs), though they still required combination use with interferon and ribavirin. ${ }^{4}$

By 2013, the U.S. Food and Drug Administration's (FDA's) approval of DAA oral regimens no longer required combination with interferon; these DAA regimens involved potentially fewer side effects, SVR rates of more than 90\%, and shortened treatment durations. ${ }^{2,4}$ Although contingent on a number of factors including genotype and history of treatment failure, newer DAAs have been reported to be generally cost-effective relative to previous standards of care (i.e., peg-interferon, ribavirin) 
and are potentially cost saving if accounting for discounted drug prices. ${ }^{5-7}$ Given the increased numbers of patients who may benefit from new agents, concerns of overall affordability have emerged. $5,6,8,9$

Chhatwal et al. (2015) estimated that new DAAs will increase hepatitis $C$ drug spending by $\$ 65$ billion over 5 years versus older treatments. However, the authors also estimated $\$ 16$ billion in overall downstream costs would be offset by the use of new DAAs versus previous medications. ${ }^{5,6,8}$ Looking at long-term HCV costs over 50 years, Van Nuys et al. (2015) estimated initial treatment costs of $\$ 150$ billion, $\$ 610$ to $\$ 1,221$ billion in additional quality-adjusted life-years, and $\$ 139$ billion in saved medical costs. ${ }^{9}$ More recently, pharmaceutical manufacturers of DAA treatments have negotiated discounts of $46 \%$ or more, resulting in HCV treatment courses meeting thresholds of being considered as "high value" for health care systems by the California Technology Assessment Forum, a division of the Institute for Clinical and Economic Review..$^{10}$

With its prevalence and sequelae, CHC places a substantial economic toll on the U.S. health care system, particularly considering the disproportionate number of individuals qualifying for Medicaid. ${ }^{1}$ As a result, many Medicaid programs have restricted access to DAA treatments, limiting coverage to individuals with more advanced disease. ${ }^{11}$ As Medicaid provides health care to a large proportion of $\mathrm{CHC}$ patients, it is necessary to further understand the Medicaid $\mathrm{CHC}$ population and to measure the burden of illness and patient and clinical characteristics associated with improved outcomes. ${ }^{12}$

Various factors have been reported to be associated with poor prognosis of $\mathrm{HCV}$ treatment, including advanced liver disease and multiple comorbidities. ${ }^{3}$ Other potential predictors of treatment failure remain poorly studied, particularly in specific populations and among those receiving multiple medications with the potential to interact negatively with DAA therapies. ${ }^{13-15}$ In this context, concomitant medications classified to interact with DAAs by the University of Liverpool HEP Drug Interactions resource may help establish the interactions that potentially affect positive outcomes. ${ }^{13-16}$

The University of Liverpool HEP Drug Interactions Resource is recommended as a resource for screening drug-drug interactions (DDIs) in the American Association for the Study of Liver Diseases HCV treatment guidance. ${ }^{17}$ Therein, the objective of this study was to assess the outcomes of receiving HCV treatment and subsequent SVR based on sociodemographic, clinical, and disease characteristics and DAA DDIs in Medicaid members with diagnosed chronic HCV.

\section{Methods}

\section{Study Design Overview}

This cross-sectional historical cohort investigation examined outcomes of treatment initiation and viral clearance associated with newer HCV agents (i.e., DAAs) among state-specific, adult
Medicaid members with diagnoses of HCV from January 1, 2014-June 30, 2015. Of key interest were associations between these outcomes and demographics, general and HCV-related clinical characteristics, and severity of DDIs with DAAs. DDI severity was classified using the University of Liverpool HEP Drug Interactions resource (available online at www.hepdruginteractions.org) and rated as (1) significant (should not be coadministered); (2) potentially clinically significant (likely to require monitoring, alteration of drug dosage, or timing of administration); (3) weak (additional action/monitoring or dosage adjustment unlikely to be required); or (4) no clinically significant interaction expected. ${ }^{16}$ The Institutional Review Board for the University of Oklahoma Health Sciences Center and the Oklahoma Health Care Authority (OHCA; i.e., the administrator of the Oklahoma Medicaid program) approved this research.

\section{Data Source}

Comprehensive Medicaid utilization and claims data were reviewed to assess those treated for $\mathrm{HCV}$; these data were maintained by Pharmacy Management Consultants (PMC), a department of the University of Oklahoma College of Pharmacy that is contracted with OHCA to perform pharmacy benefit management services to Medicaid beneficiaries. Two data sources were used: (1) an administrative paid claims database for Oklahoma Medicaid and HCV management and (2) a prior authorization database for PMC's Clinical HCV management program. Notably, OHCA had initially restricted DAA use to patients with a minimum METAVIR fibrosis score of F2 and required patients to have been illicit drug- and alcohol-free in the 6 months prior to starting treatment beginning July 1 , 2014. The HCV management program sought SVR results for each approved patient.

\section{Data Inclusion and Exclusion Criteria}

Study inclusion required $\geq 2$ HCV-related medical claims during the review period of January 1, 2014-June 30, 2015, with an International Classification of Diseases, Ninth Revision, Clinical Modification (ICD-9-CM) code (070.41, 070.42, 070.44, 070.51, 070.54, 070.7, 070.71, and v02.62). We used $\geq 2$ HCV-related claims to ensure that cases were not solely for those receiving $\mathrm{HCV}$ testing alone. Also, $\geq 6$ months of continuous Medicaid eligibility was required.

\section{Study Variables}

The study's primary outcome was receipt of HCV treatment and subsequent viral clearance based on sociodemographic, clinical, and disease characteristics and DAA DDIs. A secondary outcome of treatment failure (i.e., detectable viral load at least 12 weeks after treatment) was analyzed among Medicaid beneficiaries with collected SVR data. Independent variables included demographics (i.e., age, sex, micropolitan/rural residence); HCV treatment; prior HCV treatment (i.e., with peg-interferon, 


\section{Hepatitis C Direct-Acting Antiviral Treatment Selection, Treatment Failure, and Use of Drug-Drug Interactions in a State Medicaid Program}

\section{TABLE 1 Descriptive Statistics}

\begin{tabular}{|c|c|c|c|c|c|}
\hline & $\begin{array}{l}\text { Treated } \\
(n=464)\end{array}$ & $\begin{array}{l}\text { Untreated } \\
(n=2,948)\end{array}$ & $\begin{array}{c}\text { Overall } \\
(\mathrm{n}=3,412)\end{array}$ & \multicolumn{2}{|c|}{$\begin{array}{l}\text { Treated with DAAs } \\
\text { and SVR Measured } \\
\qquad(\mathrm{n}=168)^{\mathrm{a}}\end{array}$} \\
\hline \multicolumn{6}{|l|}{ Demographics } \\
\hline Age, years, mean $\pm S D$ & $53.6 \pm 10.0^{\mathrm{b}}$ & $49.0 \pm 11.9^{b}$ & $49.6 \pm 11.8$ & \multicolumn{2}{|c|}{$53.7 \pm 10.5$} \\
\hline Female, \% (n) & $52.8^{\mathrm{c}} \quad(245)$ & $59.5^{\mathrm{c}}(1,753)$ & $58.6 \quad(1,998)$ & 47.6 & $(80)$ \\
\hline Micropolitan or rural residence, \% (n) & $(181)$ & $41.2 \quad(1,215)$ & $40.9 \quad(1,396)$ & 33.3 & $(56)$ \\
\hline \multicolumn{6}{|l|}{ Treatment details } \\
\hline Prior hepatitis C treatment ${ }^{\mathrm{d}}$, \% (n) & $12.9^{\mathrm{e}}$ & $9.4^{\mathrm{e}} \quad(276)$ & $(336)$ & 16.1 & $(27)$ \\
\hline \multicolumn{6}{|l|}{ Direct-acting antiviral regimen, \% (n) } \\
\hline Ledipasvir/sofosbuvir & $(206)$ & - & - & 26.2 & $(44)$ \\
\hline Simeprevir & $<1.0 \quad(<10)$ & - & - & $\leq 3.0$ & $(<5)$ \\
\hline Sofosbuvir + simeprevir & $(22)$ & - & - & 7.7 & (13) \\
\hline Sofosbuvir & $(210)$ & - & - & 63.7 & $(107)$ \\
\hline Ombitasvir/paritaprevir/ritonavir + dasabuvir & $(12)$ & - & - & $\leq 3.0$ & $(\leq 5)$ \\
\hline Daclatasvir & $(13)$ & - & - & & \\
\hline \multicolumn{6}{|l|}{ DAA interaction severity ${ }^{\mathrm{f}}, \%,(\mathrm{n})$} \\
\hline Weak or no clinically significant interaction expected & $(184)$ & & & 49.4 & $(83)$ \\
\hline Significant or potentially significant interaction expected & $(149)$ & & & 23.8 & $(40)$ \\
\hline \multicolumn{6}{|l|}{ Clinical status } \\
\hline Advancing liver diseaseg, \% (n) & $22.0^{\mathrm{b}} \quad(102)$ & $14.9^{\mathrm{b}} \quad(440)$ & $(542)$ & 21.4 & (36) \\
\hline Modified CCI, mean \pm SD & $3.17 \pm 2.91^{\mathrm{b}}$ & $2.66 \pm 3.05^{b}$ & $2.73 \pm 3.04$ & 3.07 & 2.61 \\
\hline Treatment failure, \% (n) & & & & 4.8 & $(8)$ \\
\hline \multicolumn{6}{|c|}{$\begin{array}{l}\text { a Any category with } n \leq 5 \text { or } \leq 1.0 \% \text { was masked to maintain patient confidentiality. } \\
{ }^{b} P<0.001 \text { between treated and untreated via independent-group t-test or chi-square test. } \\
{ }^{c} P<0.01 \text {. } \\
{ }^{d} \text { Previous treatment defined as any treatment including peg-interferon and ribavirin; seve } \\
{ }^{e} P<0.05 \text {. } \\
{ }^{{ }^{D}} \text { DAA drug interaction. } \\
{ }^{g} \text { Advancing Liver Disease defined via the CCI (Deyo-modification). }{ }^{16,17}\end{array}$} \\
\hline
\end{tabular}

ribavirin, boceprevir, telaprevir); DDI utilization; advanced liver disease diagnoses present in medical claims (i.e., cirrhosis, ascites, hepatic cancer, hepatic encephalopathy, hepatorenal syndrome, other nonalcoholic liver disease, portal hypertension, other sequelae of chronic liver disease); a modified DeyoCharlson Comorbidity Index (CCI) that excluded diseases of the liver to prevent collinearity; and University of Liverpool severity of DDI.,15-19 Severity of DDIs was only considered for medications that involved an overlap in prescription claims between the DAA and the potentially interacting medication. ${ }^{16}$

\section{Statistical Analysis}

Descriptive statistics involving proportions and measures of central tendency were performed across all variables, with unadjusted bivariate differences between those treated with DAAs versus without assessed via straightforward application of chi-square tests and t-tests. Multivariable generalized linear models (GLMs) using a binomial/Bernoulli (logistic) family and logit link were conducted for the study's 2 outcomes of interest: (1) treatment of HCV with newer DAAs (i.e., sofosbuvir, ledipasvir/sofosbuvir, sofosbuvir + simeprevir, daclatasvir, ombitasvir/ paritaprevir/ritonavir + dasabuvir, simeprevir) across overall Medicaid membership and (2) treatment failure among those utilizing DAAs with linked SVR data. ${ }^{20}$ Findings were reported as odds ratios (ORs), with values significantly $<1.0$ indicating lower odds of an outcome, ORs $=1.0$ suggesting no difference, and ORs $>1.0$ indicating higher odds of the outcome.

Robust statistical estimation was conducted using HuberWhite (heteroskedasticity-consistent) standard errors to ensure that the analysis did not violate statistical assumptions and to control for potential skew and unequal variance within the analysis. ${ }^{21,22}$ A Bayesian sensitivity analysis was also conducted for the subgroup of cases using DAAs with SVR measurements. ${ }^{23}$ An a priori alpha of 0.05 was defined for statistical significance within the frequentist GLM framework, and all analyses were conducted using SAS 9.3 (SAS Institute, Cary, NC) and STATA MP 14.2 (StataCorp, College Station, TX).

\section{Results}

\section{Descriptive Statistics}

The Oklahoma Medicaid population during the review period averaged approximately 1 million lives annually. A total of 


\begin{tabular}{l|cc}
\hline \multicolumn{1}{c|}{ TABLE 2 } & $\begin{array}{l}\text { Common Drug Interactions by } \\
\text { Therapeutic Class }\end{array}$ \\
\hline Therapeutic Class & N (\%) \\
\hline Proton pump inhibitor & $76 \quad(27.4)$ \\
\hline Benzodiazepine & 65 & $(23.5)$ \\
\hline Calcium channel blocker & 37 & $(13.4)$ \\
\hline Inhaled LABA/ICS or ICS alone & 33 & $(11.9)$ \\
\hline Antiretroviral & 14 & $(5.1)$ \\
\hline Statin & 12 & $(4.3)$ \\
\hline Immunosuppressant & 10 & $(3.6)$ \\
\hline Opioid agonist/antagonist & 10 & $(3.6)$ \\
\hline Oral contraceptive & 9 & $(3.3)$ \\
\hline Antiarrhythmic & 4 & $(1.4)$ \\
\hline Antifungal & 4 & $(1.4)$ \\
\hline Antiepileptic & 3 & $(1.1)$ \\
\hline
\end{tabular}

ICS =inhaled corticosteroid; LABA = long-acting beta2-agonist.

3,412 patients met the study's inclusion criteria, consisting of 464 who used newer DAAs and 2,948 members who had not yet received newer HCV treatments. On average, members were $49.6 \pm 11.8$ years of age, were female $(58.6 \% ; n=1,998)$, and had urban residence $(59.1 \% ; n=2,016)$. Unadjusted analyses indicated that members who received newer DAA treatments ( $n=464)$ were significantly older, were predominantly male, had higher a proportion of prior treatments with any previous regimen and advanced liver disease, and had a higher overall CCI $(P<0.05)$. The majority of DAA cases received sofosbuvir (45.3\%) or ledipasvir/sofosbuvir (44.4\%), summing to $94.4 \%$ receiving a sofosbuvir-based regimen. Among those treated with DAAs, 39.7\% had DDIs classified as "weak" or "no clinically significant" interaction ( $\mathrm{n}=184)$, while $32.1 \%$ had interactions classified as "significant" or "potentially significant" $(n=149)$. Table 1 presents the full descriptive statistics of members meeting inclusion criteria.

Of the 464 cases who were treated, only 168 cases had a reported SVR, with 8 involving treatment failure. Overall, a majority of those with SVRs were male $(52.4 \% ; n=88)$ and resided in urban/metropolitan areas $(66.7 \% ; \mathrm{n}=112)$. Most were treated with sofosbuvir-based regimens $(63.7 \%$; $n=107)$ and had "weak" or "no clinically significant" interactions (49.4\%; n=83; Table 1).

\section{Common DDls}

The most common therapeutic classes involved in DDIs with DAA regimens were proton pump inhibitors (27.4\%), benzodiazepines (23.5\%), and calcium channel blockers (13.4\%; Table 2). Ledipasvir/sofosbuvir had the highest occurrence of "potentially clinical significant" interactions $(n=48)$ followed by sofosbuvir/simeprevir $(n=18)$. Simeprevir and ombitasvir/paritaprevir/ritonavir/dasabuvir each had 2, while sofosbuvir and sofosbuvir/daclatasvir each had 1. Significant

\begin{tabular}{l|c|c|c|c}
\multicolumn{1}{c|}{ TABLE 3 } & \multicolumn{4}{c}{$\begin{array}{l}\text { Multivariable Analysis of Chronic } \\
\text { Hepatitis C Treatment Initiation with } \\
\text { DAAs Among Medicaid Members }\end{array}$} \\
$\begin{array}{l}\text { Outcome: DAA } \\
\text { Treatment Initiation }\end{array}$ & OR & Robust SE & P Value & $95 \%$ CI \\
\hline Age & $1.037^{\mathrm{b}}$ & 0.005 & $<0.001$ & $1.026-1.048$ \\
\hline Female & 0.983 & 0.104 & 0.872 & $0.799-1.210$ \\
\hline $\begin{array}{l}\text { Micropolitan or rural } \\
\text { residence }\end{array}$ & 0.977 & 0.101 & 0.821 & $0.797-1.197$ \\
\hline Prior treatment & $1.384^{\mathrm{c}}$ & 0.214 & 0.036 & $1.022-1.874$ \\
\hline Modified CCI score & 0.994 & 0.016 & 0.730 & $0.963-1.027$ \\
\hline Advancing liver disease & $1.505^{\mathrm{d}}$ & 0.194 & 0.002 & $1.169-1.937$ \\
\hline
\end{tabular}

${ }^{a}$ Generalized linear model, binomial/Bernoulli (logistic) regression with logit link,

Huber-White heteroscedasticity consistent SEs. Log pseudo-likelihood $=-1316.893$.

$N=3,412$

${ }^{b} P<0.001$.

${ }^{c} P<0.05$.

$d P<0.01$.

$C C I=$ Charlson Comorbidity Index; $C I=$ confidence interval; $D A A=$ direct-acting antiviral; $O R=$ odds ratio; $S E=$ standard error.

interactions were limited, with sofosbuvir/simeprevir having 1 interaction and sofosbuvir alone and ombitasvir/paritaprevir/ ritonavir/dasabuvir having 3 each.

\section{Multivariable Analysis: Treatment Initiation of Chronic HCV with DAAs}

The multivariable analysis (Table 3) indicated that both prior treatment and advancing liver disease were significantly and independently associated with higher odds of treatment initiation after controlling for other clinical and demographic factors (OR for prior treatment $=1.384 ; 95 \%$ confidence interval $[C I]=1.022-1.874 ;$ OR for advancing liver disease $=1.505 ; 95 \%$ $\mathrm{CI}=1.069-1.937 ; \mathrm{P}<0.05)$. Additionally, increasing age was associated with higher odds of beginning treatment (OR for age $=1.037 ; 95 \% \mathrm{CI}=1.026-1.048 ; P<0.001)$.

\section{Multivariable Analysis: Treatment Failure Based on SVR}

Among 168 members who utilized DAAs and had subsequent SVR information reported, multivariable analysis in Table 4 indicated DDIs classified as significant or potentially significant were associated with 3.218 times higher odds of treatment failure $(95 \% \mathrm{CI}=1.584-6.530 ; P=0.001)$. Prior treatment failure was associated with 16.992 times higher odds of failure, though with a wide CI accompanying the estimation (95\% CI=2.057-140.376; $P=0.009$ ). The Bayesian sensitivity analysis (Table 5) yielded similar posterior odds concerning the significant and potentially significant interactions (posterior odds for Liverpool 3 or $4=2.238$; $95 \%$ credible interval $=1.127$ 4.556), while noting significance with interactions classified as weak or not clinically significant (posterior odds for Liverpool 1 or $2=1.362 ; 95 \%$ credible interval $=1.001-3.982$ ). Advancing liver disease not significant with Bayesian analysis 


\begin{tabular}{|c|c|c|c|c|}
\hline TABLE 4 & $\begin{array}{l}\text { tivaria } \\
\text { tment } \\
\text { licaid } \\
\text { eiving }\end{array}$ & $\begin{array}{l}\text { e Regre } \\
\text { =ailure B } \\
\text { embers } \\
\mathrm{AAs}^{\mathrm{a}}\end{array}$ & $\begin{array}{l}\text { ion } \mathrm{Ar} \\
\text { sed on } \\
\text { vith } \mathrm{He}\end{array}$ & $\begin{array}{l}\text { yses of } \\
\text { VR Among } \\
\text { titis C }\end{array}$ \\
\hline $\begin{array}{l}\text { Outcome: } \\
\text { Treatment Failure }\end{array}$ & OR & Robust SE & $P$ Value & $95 \% \mathrm{CI}$ \\
\hline Age & 1.010 & 0.070 & 0.883 & $0.882-1.157$ \\
\hline Female & \multicolumn{4}{|c|}{ Omitted $^{b}$} \\
\hline $\begin{array}{l}\text { Micropolitan or rural } \\
\text { residence }\end{array}$ & 3.307 & 3.038 & 0.193 & $0.546-20.012$ \\
\hline Prior treatment & $16.992^{c}$ & 18.306 & 0.009 & $2.057-140.376$ \\
\hline Modified CCI score & 0.781 & 0.140 & 0.168 & $0.550-1.110$ \\
\hline Advancing liver disease & 0.259 & 0.304 & 0.255 & $0.023-2.725$ \\
\hline $\begin{array}{l}\text { Weak or no clinically } \\
\text { significant interaction } \\
\text { expected }\end{array}$ & 0.667 & 0.970 & 0.780 & $0.038-11.537$ \\
\hline $\begin{array}{l}\text { Significant or } \\
\text { potentially significant } \\
\text { interaction }\end{array}$ & $3.218^{\mathrm{d}}$ & 1.161 & 0.001 & $1.587-6.530$ \\
\hline
\end{tabular}

${ }^{a}$ Generalized linear model, binomial/Bernoulli (logistic) distribution with logit link (logistic regression), maximum likelihood estimation with Huber White heteroscedasticity robust SE calculation. Log pseudo-likelihood $=-16.846 . N=168$.

${ }^{b}$ Perfect predictor: all female cases were treatment successes.

${ }^{c} P<0.01$

${ }^{d} P \leq 0.001$

$C C I=$ Charlson Comorbidity Index; $C I=$ confidence interval; $D A A=$ direct-acting antiviral; $O R=$ odds ratio; $S E=$ standard error; $S V R=$ sustained virologic response.

(posterior odds $=3.095 ; 95 \%$ credible interval $=0.412-11.296$ ), while advancing age and female sex were associated with a lower odds of treatment failure (posterior odds for age $=0.921$; 95\% credible interval $=0.851-0.984$; posterior odds for female $=0.002 ; 95 \%$ credible interval $=0.001-0.012$ ). Notably, the acceptance rate of the sensitivity analysis was relatively low $(9.5 \%)$, as was its efficiency $(<0.01 \%)$.

A subanalysis was conducted adding adherence (defined as medication possession ratio $[\mathrm{MPR}] \geq 80 \%$ ) as a covariate to the overall multivariable analysis involving cases wherein treatment durations were verified directly from prescriber practices $(n=59)$. Multivariable results were consistent in terms of significance, direction, and magnitude, with adjusted adherent cases associated with an $86.8 \%$ lower odds of treatment failure (OR for adherent $=0.132 ; 95 \% \mathrm{CI}=0.020-0.874 ; \quad P=0.036$ ). Bayesian analyses, however, did not indicate a significant association with treatment failure and MPR $\geq 80 \%$ (posterior odds for adherent $=0.303 ; 95 \%$ credible interval $=0.003-1.660$ ) .

\section{Discussion}

This study combined clinical laboratory and administrative claims data to assess outcomes of treatment initiation with DAAs and subsequent treatment failure among adult members of a state Medicaid program with HCV according to demographics, clinical factors, and DDIs. Overall, treatment success was high, and DDIs were present in a substantial portion of

\begin{tabular}{|c|c|c|c|c|}
\hline \multicolumn{5}{|c|}{$\begin{array}{l}\text { Bayesian Sensitivity Analysis, } \\
\text { Multivariable Regression of Treatment } \\
\text { Failure Based on SVR Among Medicaid } \\
\text { Members with Hepatitis C Receiving } \\
\text { DAAsa }\end{array}$} \\
\hline $\begin{array}{l}\text { Outcome: } \\
\text { Treatment Failure }\end{array}$ & $\begin{array}{l}\text { Posterior } \\
\text { OR Mean }\end{array}$ & SD & MCSE & $\begin{array}{l}95 \% \text { Credible } \\
\text { Interval }\end{array}$ \\
\hline Age & 0.921 & 0.033 & 0.001 & $0.851-0.984$ \\
\hline Female & 0.002 & 0.005 & 0.001 & $0.001-0.012$ \\
\hline $\begin{array}{l}\text { Micropolitan or rural } \\
\text { residence }\end{array}$ & 1.119 & 1.147 & 0.034 & $0.074-4.590$ \\
\hline Prior treatment & 4.643 & 5.893 & 0.192 & $0.280-18.769$ \\
\hline Modified CCI score & 1.016 & 0.223 & 0.007 & $0.662-1.471$ \\
\hline Advancing liver disease & 3.095 & 3.147 & 0.096 & $0.412-11.296$ \\
\hline $\begin{array}{l}\text { Weak or no clinically } \\
\text { significant interaction } \\
\text { expected }\end{array}$ & 1.362 & 2.315 & 0.052 & $1.001-3.982$ \\
\hline $\begin{array}{l}\text { Significant or } \\
\text { potentially significant } \\
\text { interaction }\end{array}$ & 2.238 & 0.934 & 0.026 & $1.127-4.556$ \\
\hline
\end{tabular}

a Bayesian binomial (logistic) regression, random-walk Metropolis-Hastings sampling, Markov chain Monte Carlo sample size 500,000, burn-in 150,000, noninformative prior, acceptance rate $=9.5 \%$, efficiency $<0.01 . N=168$.

$C C I=$ Charlson Comorbidity Index; DAA = direct-acting antiviral; $M C S E=$ Monte Carlo Standard Error; $O R=$ odds ratio; $S E=$ standard error; $S V R=$ sustained virologic response.

cases. A higher odds of treatment initiation was associated with increasing age, prior HCV treatment, and advanced liver disease. As the state Medicaid program at that time focused on the population with METAVIR scores of F2 or above, this finding may be largely related to the policy. Based on the University of Liverpool DDI classifications, significantly higher odds of treatment failure were also associated with significant or potentially significant DDIs. Overall, this work among Medicaid beneficiaries sought to extend other studies involving underserved or vulnerable populations in an effort to provide insight into demographic and clinical profiles of those being chosen for treatment and to highlight potential associations of concomitant drug usage with treatment failure in a real-world Medicaid population setting. 2,4,22,23,27

Previous research using the Liverpool DDI classification system has not necessarily observed a relationship between treatment failure according to SVR and DDIs. ${ }^{22,23}$ In evaluating 300 patients in a veteran population, Ottman et al. (2018) did not report an association between achievement of SVR and presence of DDIs according to the Liverpool classification, though the study was noted to be underpowered to discern between DDI severity groupings. The majority of DDIs identified in Ottman et al. (2018) were categorized as significant or potentially clinically significant $(76 \%)$, whereas the current observed substantially fewer (28.5\%). ${ }^{24}$ These authors found a similar percentage with DDIs as the present study with no clinically significant DDIs (80.3\% vs. $71.8 \%){ }^{24}$ Vermehren 
et al. (2016) also evaluated the Liverpool DDI classification in a retrospective study of $541 \mathrm{HCV}$-treated patients. ${ }^{25}$ Results indicated a similar percentage of patients found to have potentially significant interactions (35.0\% vs. $32.1 \%$ ); no evaluation of SVR outcomes was undertaken, however..$^{25}$

Among other findings in the present investigation, multivariable findings indicated that advanced liver disease was associated with 50.5\% higher odds of DAA treatment initiation $(P=0.002)$; this observation likely reflects the state Medicaid program requirement of METAVIR fibrosis score of F2 or higher for treatment approval beginning on July 1, 2014. Potentially, this may also reflect a prescriber's choice to treat or attributes of a patient seeking care. However, if it is deemed that a cure is urgently needed for patients with more advanced sequelae, then those with advanced liver disease may have been prioritized. The $+38.4 \%$ higher odds of DAA treatment initiation associated with previous treatment experience for HCV may have been observed for several reasons, including remaining at a higher level of care for HCV or, again, a willingness to seek a cure for their HCV status.

SVR information was available for less than half of the study population $(36.2 \%$; 168 of 464). This information was collected via the state Medicaid program for patients approved for treatment on a voluntary basis from the prescribing practitioner. Descriptive information indicated that the treated population with available SVRs was generally similar to those without, though the type of treatment regimens appeared to differ. However, given that SVRs were collected 12 weeks after completion of treatment and because sofosbuvir-only regimens were utilized earlier in the review period (i.e., ledipasvir/sofosbuvir became available in 2015), more patients with reported SVRs were likely to be on sofosbuvir-only regimens. Future research should recognize that SVR success rates may ultimately differ based on the regimen used.

The presence of any DDI (among those with a reported SVR) at any time during the study is a notable finding (73.2\%), irrespective of severity. While a smaller percentage of cases involving significant or potentially significant DDIs was present, these were associated with an approximately 3 -fold higher odds of treatment failure, with results of a Bayesian sensitivity analysis supporting these initial findings. Continued work should be sought to assess prescribers' knowledge of these DDIs, including methods to mitigate potential DDIs. ${ }^{26}$

\section{Limitations}

Despite findings, certain limitations of this work should be considered. Due to the prior authorization criteria and program engagement, the cases included occurred during a period wherein more active engagement to treat certain cases may have been present within the state Medicaid program. Therein, a selection bias may have been present that could not be captured within this analysis and may affect the interpretation or generalizability of findings. Consistent with many Medicaid programs, the SVR data collected were not routine; these could only be collected voluntarily and via a designated clinical management program. As such, all persons treated with DAAs could not be assessed for treatment success or failure based on SVR, and it cannot be ascertained if those without reported SVRs experienced different rates of treatment success.

As the current work was undertaken across a single state Medicaid program spanning from January 1, 2014-June 30, 2015, caution should be exercised in generalizing results to other patient populations or time frames, given that other states may differ in eligibility and protocols, particularly over time. Prior authorizations of the aforementioned newer DAA therapies were not implemented until July 1, 2014; before prior authorization, patients were not monitored for guideline-recommended regimens including concomitant use with ribavirin and interferon. After July 1, 2014, prior authorizations were not approved without use of appropriate concomitant therapy. Concomitant therapy with ribavirin and interferon was not assessed in this study and could affect SVR outcomes.

Finally, no long-term follow-up was undertaken concerning disease monitoring and hepatocellular cancer screening, despite recommendations to do so. ${ }^{25,26} \mathrm{Kim}$ et al. (2018) recognized the lack of research surrounding post-SVR treatment success, particularly involving that which identified gaps in underserved or vulnerable populations; these researchers found a significantly worse continuum of care among patients with advanced fibrosis after HCV treatment success. ${ }^{27}$

\section{Conclusions}

$\overline{\text { This investigation of Medicaid beneficiaries with HCV found }}$ a higher odds of treatment initiation with advancing age, previous HCV treatment attempts, and advanced liver disease. While findings may reflect the prior authorization criteria in place, these criteria were not limited strictly to advanced HCV patients; physicians may have prioritized more advanced cases to be treated first. Treatment success based on SVR was generally high, although those with DDIs classified as significant or potentially significant were associated with a markedly higher odds of treatment failure. Continued research is warranted to further elicit the relationship between potential DDIs and DAA-based therapy on outcomes in HCV.

\section{Authors}

SHELLIE L. KEAST, PharmD, PhD; BETHANY HOLDERREAD, PharmD; TERRY COTHRAN, DPh; and GRANT H. SKREPNEK, $\mathrm{PhD}$, University of Oklahoma College of Pharmacy, Oklahoma City.

AUTHOR CORRESPONDENCE: Shellie L. Keast, PharmD, PhD, Assistant Professor, University of Oklahoma College of Pharmacy, CPB-222, P.O. Box 26901, Oklahoma City, OK 73126-0901. Tel.: 405.271.8222; E-mail: shellie-keast@ouhsc.edu. 


\section{Hepatitis C Direct-Acting Antiviral Treatment Selection, Treatment Failure, and Use of Drug-Drug Interactions in a State Medicaid Program}

\section{DISCLOSURES}

This research was funded by an unrestricted research grant by Gilead Sciences. During the course of this study, all authors were either employed by the Oklahoma HealthCare Authority or engaged in contractual work for this employer. Keast, Holderread, and Skrepnek report unrelated research grants from AbbVie, Otsuka, and Amgen. Keast and Skrepnek acknowledge funding from Purdue Pharma for an unrelated research fellowship grant.

Posters based on this work were presented at HepDart 2015 on December 6-10, 2015, in Grand Wailea, HI, and at Academy of Managed Care Nexus 2015 on October 26-29, 2015, in Orlando, FL.

\section{REFERENCES}

1. Denniston MM, Jiles RB, Drobeniuc J, et al. Chronic hepatitis C virus infection in the United States, National Health and Nutrition Examination Survey 2003 to 2010. Ann Intern Med. 2014;160(5):293-300.

2. Centers for Disease Control and Prevention. Hepatitis $C$ questions and answers for healthcare professionals. 2015. Available at: www.cdc. gov/hepatitis/hcv/hcvfaq.htm. Accessed October 6, 2019.

3. American Association for the Study of Liver Diseases and Infectious Diseases Society of American HCV Guidance Panel. Hepatitis C guidance: AASLD-IDSA recommendations for testing, managing, and treating adults infected with hepatitis C virus. Hepatology. 2015;62(3):932-54.

4. Franciscus A. HCSP fact sheet: a brief history of hepatitis C. 2015. Available at: https://npin.cdc.gov/publication/hcsp-fact-sheet-brief-historyhepatitis-c. Accessed October 16, 2019

5. Chhatwal J, Kanwal F, Roberts MS, Dunn MA. Cost-effectiveness and budget impact of hepatitis $C$ virus treatment with sofosbuvir and ledipasvir in the United States. Ann Intern Med. 2015;162(6):397-406.

6. Linas BP, Barter DM, Morgan JR, et al. The cost-effectiveness of sofosbuvirbased regimens for treatment of hepatitis $C$ virus genotype 2 or 3 infection. Ann Intern Med. 2015;162(9):619-29.

7. Chhatwal J, He T, Hur C, Lopez-Olivo MA. Direct-acting antiviral agents for patients with hepatitis $C$ virus genotype 1 infection are cost-saving. Clin Gastroenterol Hepatol. 2017;15(6):827-837.e8

8. Rein DB, Wittenborn JS, Smith BD, Liffmann DK, Ward JW. The costeffectiveness, health benefits, and financial costs of new antiviral treatments for hepatitis C virus. Clin Infect Dis. 2015;61(2):157-68.

9. Van Nuys K, Brookmeyer R, Chou JW, Dreyfus D, Dieterich D, Goldman DP. Broad hepatitis $C$ treatment scenarios return substantial health gains, but capacity is a concern. Health Aff. 2015;34(10):1666-74.

10. Institute for Clinical and Economic Review. New lower prices for Gilead hepatitis C drugs reach CTAF threshold for high health system value. 2015. Available at: https://icer-review.org/announcements/new-lower-prices-forgilead-hepatitis-c-drugs-reach-ctaf-threshold-for-high-health-system-value/. Accessed October 6, 2019.

11. Barua S, Greenwald R, Grebely J, Dore GJ, Swan T, Taylor LE. Restrictions for Medicaid reimbursement of sofosbuvir for the treatment of hepatitis $C$ virus infection in the United States. Ann Intern Med. 2015;163(3):215-23.
12. Swartz K. Health care for the poor: for whom, what care, and whose responsibility? Focus. 2009;26(2):69-74. Available at: https://www.irp.wisc. edu/publications/focus/pdfs/foc262l.pdf. Accessed October 6, 2019

13. Rice Jr DP, Faragon JJ, Banks S, Chirch LM. HIV/HCV antiviral drug interactions in the era of direct-acting antivirals. J Clin Translational Hepatol. 2016;4(3):234.

14. Zu Siederdissen CH, Maasoumy B, Marra F, et al. Drug-drug interactions with novel all oral interferon-free antiviral agents in a large real-world cohort. Clin Infect Dis. 2016;62(5):561-67.

15. Burgess S, Partovi N, Yoshida EM, Erb SR, Azalgara VM, Hussaini T. Drug interactions with direct-acting antivirals for hepatitis C: implications for HIV and transplant patients. Ann Pharmacotherapy. 2015;49(6):674-87.

16. University of Liverpool. Hep drug interactions checker. 2018. Available at: https://www.hep-druginteractions.org/. Accessed October 6, 2019.

17. AASLD-IDSA. Patients with HIV/HCV coinfection. Recommendations for testing, managing, and treating hepatitis C. 2018. Available at: https://www. hcvguidelines.org/unique-populations/hiv-hcv. Accessed October 6, 2019.

18. Charlson M, Pompei P, Ales K, MacKenzie R. A new method of classifying prognostic comorbidity in longitudinal studies: development and validation. J Chronic Dis. 1987;40(5):373-83.

19. Deyo R, Cherkin D, Ciol M. Adapting a clinical comorbidity index for use with ICD-9-CM administrative databases. J Clin Epidemiol. 1992;45(6):613-19.

20. Skrepnek GH, Olvey EL, Sahai A. Econometric approaches in evaluating costs and outcomes within pharmacoeconomic analyses. Pharm Policy Law. 2012;14(1):105

21. Huber P. The behavior of maximum likelihood estimates under nonstandard conditions. Proceedings of the Fifth Berkeley Symposium on Mathematical Statistics and Probability. Berkeley and Los Angeles: University of California Press; 1967;1:221-33.

22. White H. A heteroskedasticity-consistent covariance matrix estimator and a direct test for heteroskedasticity. Econometrica. 1980;48(4):817-38.

23. Skrepnek GH. The contrast and convergence of Bayesian and frequentist statistical approaches in pharmacoeconomic analysis. Pharmacoeconomics. 2007;25(8):649-64

24. Ottman AA, Townsend ML, Hashem MG, DiMondi VP, Britt RB. Incidence of drug interactions identified by clinical pharmacists in veterans initiating treatment for chronic hepatitis $\mathrm{C}$ infection. Ann Pharmacother 2018;52(8):763-68.

25. Vermehren J, Peiffer KH, Welsch C, et al. The efficacy and safety of direct acting antiviral treatment and clinical significance of drug-drug interactions in elderly patients with chronic hepatitis $\mathrm{C}$ virus infection. Aliment Pharmacol Ther. 2016;44(8):856-65.

26. Talavera Pons S, Boyer A, Lamblin G, et al. Managing drug-drug interactions with new direct-acting antiviral agents in chronic hepatitis C. Br J Clin Pharmacology. 2017:83(2):269-93. 